PROCEEDINGS OF THE

AMERICAN MATHEMATICAL SOCIETY

Volume 30, No. 1, September 1971

\title{
BOUNDS FOR THE DETERMINANT OF THE SUM OF HERMITIAN MATRICES ${ }^{1}$
}

\author{
MIROSLAV FIEDLER
}

Abstract. Best possible lower and upper bounds for the determinant of the sum of two hermitian matrices in terms of the eigenvalues of both matrices are obtained.

It is the main purpose of this note to prove the following

TheOREM. Let $A$ and $B$ be hermitian $n \times n$ matrices with eigenvalues $\alpha_{1} \geqq \alpha_{2} \geqq \cdots \geqq \alpha_{n}$ and $\beta_{1} \geqq \beta_{2} \geqq \cdots \geqq \beta_{n}$ respectively. Then

$$
\min _{P} \prod_{i=1}^{n}\left(\alpha_{i}+\beta_{P i}\right) \leqq \operatorname{det}(A+B) \leqq \max _{P} \prod_{i=1}^{n}\left(\alpha_{i}+\beta_{P i}\right)
$$

(the minimum or maximum is taken over all permutations of indices $1,2, \cdots, n)$.

In particular, if $\alpha_{n}+\beta_{n} \geqq 0$ (which is certainly true if both $A$ and $B$ are positive semidefinite) then

$$
\prod_{i=1}^{n}\left(\alpha_{i}+\beta_{i}\right) \leqq \operatorname{det}(A+B) \leqq \prod_{i=1}^{n}\left(\alpha_{i}+\beta_{n+1-i}\right) .
$$

These estimates are best possible in terms of the eigenvalues of $A$ and $B$.

Proof. Let us first prove the

Lemma. Let $P$ and $Q$ be complex $n \times n$ matrices, $\operatorname{det} P \neq 0$. Then for any complex $\epsilon$ sufficiently small in modulus,

$$
\operatorname{det}(P+\epsilon Q)=\operatorname{det} P\left(1+\epsilon \operatorname{tr} Q P^{-1}\right)+O\left(\epsilon^{2}\right) .
$$

REMARK. As usual, $\operatorname{tr} Z$ means the trace, $\sum z_{i i}$, of the square matrix $Z=\left(z_{i k}\right)$. We shall of ten use the formula

$$
\operatorname{tr} A B=\operatorname{tr} B A \text {. }
$$

Proof of the Lemma. If $P=I$, the identity matrix, (3) is immediate. Then it suffices to use this for the second term in $\operatorname{det}(P+\epsilon Q)$ $=\operatorname{det} P \operatorname{det}\left(I+\epsilon Q P^{-1}\right)$.

Received by the editors September 1, 1970.

AMS 1969 subject classifications. Primary 1558.

Key words and phrases. Determinant, hermitian matrices.

${ }^{1}$ This paper was prepared while the author was a visiting professor at Auburn University under the National Science Foundation Senior Foreign Fellowship Grant in 1969-70. 
Now, let $A$ and $B$ be hermitian matrices with eigenvalues $\alpha_{1} \geqq \cdots \geqq \alpha_{n}$ and $\beta_{1} \geqq \cdots \geqq \beta_{n}$, respectively.

Let us prove the left inequality in (1) first under the assumption that $\alpha_{1}>\alpha_{2}>\cdots>\alpha_{n}$.

The matrix $A$ is unitarily similar to $A_{0}=\operatorname{diag}\left\{\alpha_{1}, \alpha_{2}, \cdots, \alpha_{n}\right\}$ and $B$ is unitarily similar to $B_{0}=\operatorname{diag}\left\{\beta_{1}, \beta_{2}, \cdots, \beta_{n}\right\}$. Clearly, $\operatorname{det}(A+B)=\operatorname{det}\left(A_{0}+V B_{0} V^{*}\right)$ for some unitary matrix $V$.

The set $U_{n}$ of all unitary $n \times n$ matrices being compact, there exists a matrix $V_{0} \in U_{n}$ such that

$$
\operatorname{det}\left(A_{0}+V_{0} B_{0} V_{0}^{*}\right)=\min _{V \in U_{n}} \operatorname{det}\left(A_{0}+V B_{0} V^{*}\right) .
$$

Denote

$$
\begin{aligned}
& B_{1}=V_{0} B_{0} V_{0}^{*}, \\
& C_{0}=A_{0}+V_{0} B_{0} V_{0}^{*},
\end{aligned}
$$

so that clearly

$$
\operatorname{det} C_{0} \leqq \operatorname{det}(A+B) \text {. }
$$

Let us prove that if $C_{0}$ is nonsingular then $C_{0}$ commutes with $B_{1}$ :

$$
B_{1} C_{0}=C_{0} B_{1} \text {. }
$$

Assume that (8) is not fulfilled. Then the skew-hermitian matrix $S=C_{0}^{-1} B_{1}-B_{1} C_{0}^{-1} \neq 0, S=-S^{*}$, so that

$$
\operatorname{tr} S\left(B_{1} C_{0}^{-1}-C_{0}^{-1} B_{1}\right)=\operatorname{tr} S S^{*}>0 .
$$

For any real $\epsilon$, the matrix $V(\epsilon)=\exp \epsilon S=I+\epsilon S+\frac{1}{2} \epsilon^{2} S^{2}+\cdots$ is unitary. Choose now $\epsilon$ small enough in modulus such that

$$
\epsilon \operatorname{det} C_{0}<0 \text {. }
$$

Then, according to the Lemma,

$$
\begin{aligned}
\operatorname{det}\left(A_{0}+V(\epsilon) B_{1} V^{*}(\epsilon)\right) & =\operatorname{det}\left(A_{0}+B_{1}+\epsilon\left(S B_{1}-B_{1} S\right)\right)+O\left(\epsilon^{2}\right) \\
& =\operatorname{det} C_{0}\left(1+\epsilon \operatorname{tr}\left(S B_{1}-B_{1} S\right) C_{0}^{-1}\right)+O\left(\epsilon^{2}\right) \\
& =\operatorname{det} C_{0}\left(1+\epsilon \operatorname{tr} S\left(B_{1} C_{0}^{-1}-C_{0}^{-1} B_{1}\right)\right)+O\left(\epsilon^{2}\right)
\end{aligned}
$$

which is, for sufficiently small $\epsilon$ in modulus, less than $\operatorname{det} C_{0}$ by (9) and (10).

This contradiction proves (8). By (5) and (6), $B_{1}\left(A_{0}+B_{1}\right)$ $=\left(A_{0}+B_{1}\right) B_{1}$ or $A_{0} B_{1}=B_{1} A_{0}$.

Since $A_{0}$ is diagonal with distinct diagonal entries, $B_{1}$ is easily seen to be diagonal as well. It follows that for some permutation $P_{0}$, 
$B_{1}=\operatorname{diag}\left\{\beta_{P_{0} 1}, \beta_{P_{0} 2}, \cdots, \beta_{P_{0} n}\right\}$ so that by (7), $\prod_{i}\left(\alpha_{i}+\beta_{P_{0} i}\right)$ $\leqq \operatorname{det}(A+B)$, and the left-hand side is clearly equal to $\min _{P} \prod_{i}\left(\alpha_{i}+\beta_{P i}\right)$.

Let us now show that the result is still valid if $\operatorname{det} C_{0}=0$ and/or if we drop the assumption that the eigenvalues $\alpha_{1}, \cdots, \alpha_{n}$ of $A$ are mutually distinct. The dependence of $\operatorname{det} C_{0}$ on $A$ is easily seen to be continuous with no local extreme points. Hence we can construct a sequence $\left\{A_{k}\right\}$ of hermitian matrices with distinct roots converging to $A$ and such that the corresponding matrices $C_{0 k}$ from (6) are all nonsingular. The eigenvalues $\alpha_{k 1} \geqq \alpha_{k 2} \geqq \cdots \geqq \alpha_{k n}$ of $A_{k}$ will then converge, $\alpha_{k j} \rightarrow \alpha_{j}, j=1, \cdots, n$, so that the left inequality in (1) will be satisfied also for the limit.

The proof of the right inequality in (1) is similar; the sign of $\epsilon$ will then be chosen the same as the sign of the matrix for which the maximum is attained.

The inequalities (2) follow from (1). If $\alpha_{n}+\beta_{n} \geqq 0$ then $\alpha_{i}+\beta_{j} \geqq 0$ for all $i, j$ and clearly

$$
\begin{aligned}
\prod_{i}\left(\alpha_{i}+\beta_{i}\right) & =\min _{P} \prod_{i}\left(\alpha_{i}+\beta_{P i}\right), \\
\prod_{i}\left(\alpha_{i}+\beta_{n+1-i}\right) & =\max _{P} \prod_{i}\left(\alpha_{i}+\beta_{P i}\right)
\end{aligned}
$$

since, for $i<j$ and $i^{\prime}<j^{\prime}$,

$$
\left(\alpha_{i}+\beta_{i^{\prime}}\right)\left(\alpha_{j}+\beta_{j^{\prime}}\right)-\left(\alpha_{i}+\beta_{j^{\prime}}\right)\left(\alpha_{j}+\beta_{i^{\prime}}\right)=-\left(\alpha_{i}-\alpha_{j}\right)\left(\beta_{i^{\prime}}-\beta_{j^{\prime}}\right) \leqq 0
$$

and every permutation can be expressed as a product of transpositions. The proof is complete.

Let us add some remarks. First, the inequalities in (1) and the left inequality in (2) can be generalized to the case of more than two hermitian matrices. In particular, it follows that for any $x$ real,

$$
\min _{P} \prod_{i}\left(\alpha_{i}+\beta_{P i}+x\right) \leqq \operatorname{det}(A+B+x I) \leqq \max _{P} \prod_{i}\left(\alpha_{i}+\beta_{P i}+x\right) \text {. }
$$

Similarly, for any $x$ such that $x+\alpha_{n}+\beta_{n} \geqq 0$,

$$
\prod_{i}\left(\alpha_{i}+\beta_{i}+x\right) \leqq \operatorname{det}(A+B+x I) \leqq \prod_{i}\left(\alpha_{i}+\beta_{n+1-i}+x\right),
$$

and, for any $x$ satisfying $x \geqq \alpha_{1}+\beta_{1}$, we have

$$
\prod_{i}\left(x-\alpha_{i}-\beta_{i}\right) \leqq \operatorname{det}(x I-A-B) \leqq \prod_{i}\left(x-\alpha_{i}-\beta_{n+1-i}\right) .
$$

Let us denote by $E_{k}\left(x_{1}, \cdots, x_{n}\right)$ the $k$ th elementary symmetric function of $x_{1}, \cdots, x_{n}$. If $\gamma_{1}, \cdots, \gamma_{n}$ are eigenvalues of $C=A+B$, 
it follows easily, by taking $x$ very large, that, for any hermitian $A$ and $B$,

$$
\begin{aligned}
E_{2}\left(\alpha_{1}+\beta_{n}, \alpha_{2}+\beta_{n-1}, \cdots, \alpha_{n}+\beta_{1}\right) & \geqq E_{2}\left(\gamma_{1}, \cdots, \gamma_{n}\right) \\
& \geqq E_{2}\left(\alpha_{1}+\beta_{1}, \cdots, \alpha_{n}+\beta_{n}\right) .
\end{aligned}
$$

Since $E_{1}\left(\gamma_{1}, \cdots, \gamma_{n}\right)=E_{1}\left(\alpha_{1}+\beta_{1}, \cdots, \alpha_{n}+\beta_{n}\right)$, the last inequality is equivalent to $\sum_{i} \gamma_{i}^{2} \leqq \sum_{i}\left(\alpha_{i}+\beta_{i}\right)^{2}$ which is easily seen (the lefthand side is $\operatorname{tr}(A+B)^{2}$ etc.) to be equivalent to von Neumann's inequality $\operatorname{tr} A B \leqq \sum_{i} \alpha_{i} \beta_{i}$.

Let us show that the left inequality in (2) can be derived from a result by Lidskii [3], Wielandt [4]: The intersection of the convex hull of points $\left(\alpha_{1}+\beta_{P 1}, \alpha_{2}+\beta_{P 2}, \cdots, \alpha_{n}+\beta_{P n}\right)$ with the convex hull of points $\left(\beta_{1}+\alpha_{P 1}, \beta_{2}+\alpha_{P 2}, \cdots, \beta_{n}+\alpha_{P n}\right)$ contains the point $\left(\gamma_{1}, \cdots, \gamma_{n}\right)$. If $\alpha_{n}+\beta_{n} \geqq 0$, all these points are in the nonnegative orthant where the region

$$
\prod_{i=1}^{n} x_{i} \geqq \min _{P} \prod_{i}\left(\alpha_{i}+\beta_{P i}\right)=\prod_{i}\left(\alpha_{i}+\beta_{i}\right)
$$

is convex. Hence $\operatorname{det}(A+B)=\prod_{i=1}^{n} \gamma_{i} \geqq \prod_{i}\left(\alpha_{i}+\beta_{i}\right)$.

Since in the nonnegative orthant the region

$$
\begin{aligned}
E_{k}\left(x_{1}, \cdots, x_{n}\right) & \geqq \min _{P} E_{k}\left(\alpha_{1}+\beta_{P 1}, \alpha_{2}+\beta_{P 2}, \cdots, \alpha_{n}+\beta_{P n}\right) \\
& =E_{k}\left(\alpha_{1}+\beta_{1}, \alpha_{2}+\beta_{2}, \cdots, \alpha_{n}+\beta_{n}\right)
\end{aligned}
$$

is convex for each $k=1,2, \cdots, n$, the same argument shows that, if $x_{n}+\beta_{n} \geqq 0$, then, for $k=1, \cdots, n$,

(11) $E_{k}\left(\gamma_{1}, \gamma_{2}, \cdots, \gamma_{n}\right) \geqq E_{k}\left(\alpha_{1}+\beta_{1}, \alpha_{2}+\beta_{2}, \cdots, \alpha_{n}+\beta_{n}\right)$.

This is of course also the best possible estimate for the sum of all principal minors of order $k$ of the matrix $A+B$ in terms of the eigenvalues of the (hermitian) matrices $A$ and $B$.

The same argument applied to the functions $Q_{k}\left(x_{1}, \cdots, x_{n}\right)$ $=E_{k}\left(x_{1}, \cdots, x_{n}\right) / E_{k-1}\left(x_{1}, \cdots, x_{n}\right)(k=2, \cdots, n)$ which are also convex in the nonnegative orthant (cf. [1]) shows that even

$$
\begin{aligned}
Q_{k}\left(\gamma_{1}, \cdots, \gamma_{n}\right) & \geqq \min _{P} Q_{k}\left(\alpha_{1}+\beta_{P 1}, \cdots, \alpha_{n}+\beta_{P n}\right) \\
& =Q_{k}\left(\alpha_{1}+\beta_{1}, \cdots, \alpha_{n}+\beta_{n}\right) \text { for } k=2, \cdots, n .
\end{aligned}
$$

This last equality follows from the following assertion:

Let $P_{1}, P_{2}$ be permutations of $1,2, \cdots, n$ such that $P_{1} k \neq P_{2} k$ only for $k=i$ and $k=j, i<j$ (so that $P_{2}$ is a product of $P_{1}$ and a transposition). If $P_{1} i<P_{1} j$, then 
(13) $Q_{k}\left(\alpha_{1}+\beta_{P_{1} 1}, \cdots, \alpha_{n}+\beta_{P_{1} n}\right) \leqq Q_{k}\left(\alpha_{1}+\beta_{P_{2} 1}, \cdots, \alpha_{n}+\beta_{P_{2} n}\right)$.

To show this, we can assume $i=n-1, j=n$ and $P_{1}(n-1)<P_{1}(n)$. Then, (13) is equivalent to $\operatorname{det}\left(\begin{array}{ll}E_{k}\left(\alpha_{1}+\beta_{P_{1} 1}, \cdots, \alpha_{n}+\beta_{P_{1} n}\right) & E_{k-1}\left(\alpha_{1}+\beta_{P_{1} 1}, \cdots, \alpha_{n}+\beta_{P_{1} n}\right) \\ E_{k}\left(\alpha_{1}+\beta_{P_{2} 1}, \cdots, \alpha_{n}+\beta_{P_{2} n}\right) & E_{k-1}\left(\alpha_{1}+\beta_{P_{2} 1}, \cdots, \alpha_{n}+\beta_{P_{2} n}\right)\end{array}\right) \leqq 0$.

Using the formula

$$
\begin{aligned}
E_{k}\left(z_{1}, \cdots, z_{n}\right)= & E_{k}\left(z_{1}, \cdots, z_{n-2}\right)+\left(z_{n-1}+z_{n}\right) E_{k-1}\left(z_{1}, \cdots, z_{n-2}\right) \\
& +z_{n-1} z_{n} E_{k-2}\left(z_{1}, \cdots, z_{n-2}\right)
\end{aligned}
$$

and the notation

$$
E_{k}=E_{k}\left(\alpha_{1}+\beta_{P_{1} 1}, \cdots, \alpha_{n-2}+\beta_{P_{1}(n-2)}\right),
$$

one shows easily that the above determinant is equal to

$$
\begin{aligned}
-\left(\alpha_{n-1}-\alpha_{n}\right)\left(\beta_{P_{1}(n-1)}-\beta_{P_{1} n}\right) & {\left[\left(\alpha_{n-1}+\alpha_{n}+\beta_{P_{1}(n-1)}+\beta_{P_{1} n}\right)\right.} \\
\cdot & \left.\left(E_{k-2}^{2}-E_{k-3} E_{k-1}\right)+E_{k-2} E_{k-1}-E_{k-3} E_{k}\right]
\end{aligned}
$$

since $P_{2}(n-1)=P_{1}(n), P_{2}(n)=P_{1}(n-1)$. However, the well-known inequalities (cf. [2]) yield $E_{\mathbf{k - 2}}^{2} \geqq E_{k-3} E_{k-1}, E_{k-2} E_{k-1} \geqq E_{k-3} E_{k}$, so that the last expression is nonpositive and (13) is true.

It is easy to see that the system (12), together with $E_{1}\left(\gamma_{1}, \cdots, \gamma_{n}\right)$ $=E_{1}\left(\alpha_{1}+\beta_{1}, \cdots, \alpha_{n}+\beta_{n}\right)$ is stronger than the system (11).

\section{BIBLIOGRAPHY}

1. E. F. Beckenbach and R. Bellman, Inequalities, Ergebnisse der Mathematik und ihrer Grenzgebiete, Band 30, Springer-Verlag, Berlin, 1961. MR 28 \#1266.

2. G. H. Hardy, J. E. Littlewood and G. P6lya, Inequalities, 2nd ed., Cambridge Univ. Press, New York, 1952. MR 13, 727.

3. V. C. Lidskir, The proper values of the sum and product of symmetric matrices, Dokl. Akad. Nauk SSSR 75 (1950), 769-772. MR 12, 581.

4. H. Wielandt, An extremum property of sums of eigenvalues, Proc. Amer. Math. Soc. 6 (1955), 106-110. MR 16, 785.

Auburn University, Auburn, Alabama 36830

Czechoslovak Academy of Sciences, Prague, Czechoslovakia 